\title{
TOWARDS A CONCEPT FOR STREAMLINING GAME DESIGN OF AN EXISTING SERIOUS GAME AND PRELIMINARY EVALUATION
}

\author{
Daniel Atorf ${ }^{1}$, Ehm Kannegieser ${ }^{1}$ and Marlene Dillig ${ }^{2}$ \\ ${ }^{1}$ Fraunhofer IOSB, Fraunhoferstr. 1, 76197 Karlsruhe, Germany \\ ${ }^{2}$ Hochschule Furtwangen University, Robert-Gerwig-Platz 1, 78120 Furtwangen, Germany
}

\begin{abstract}
This paper elaborates on a process to streamline the game design of the existing serious game Lost Earth 2307. Previous evaluations of the game revealed issues in terms of usability on the one hand and new requirements, such as motivation, accessibility via web and shorter gameplay, on the other hand. Based on the findings and the new requirements, the modification of the initial game design and the methods used are presented and elaborated. Furthermore, the question whether the resulting streamlined concept provides motivation and improves on usability is discussed. A subjective evaluation of this question is shown, followed up by presenting a preliminary study on a prototype implementation. The paper depicts the setup of the study using SUS and Flow Short Scale questionnaires and reports about the results and discusses the outcomes.
\end{abstract}

\section{KEYWORDS}

Digital Game-Based Learning, Game Design, Gamification, Usability, Preliminary Study

\section{INTRODUCTION}

The serious game Lost Earth 2307 was developed for teaching on how to analyze aerial and satellite images and on how to formally report on identified objects by using specific terms. This training domain is rather complex to teach, therefore individual training courses last several months and trainers have a high interest in keeping the students motivated over a long time. The game addresses this need by following the ideas of Digital Game Based Learning (Prensky 2007) and Immersive Didactics (Bopp 2006). It is integrated into the course as an optional element in the informal learning phase where students have to intensify their knowledge.

The game was evaluated iteratively during development, focusing on the learning outcome, on game functionality and user experience (Atorf et al. 2019a). Additional on site evaluation of immersion and usability showed that the learnability of the game was an issue. A pedagogical agent was implemented in order to improve learnability. However, a study on this implementation indicated, that the usability in Lost Earth 2307 should generally be improved (Atorf et al. 2019b). Usability issues greatly interfere with immersion, thus preventing acceptance and good learning outcomes (Cheng et al. 2015).

Further on site evaluation and reviews confirmed the described findings on usability. In addition, the requirements evolved on behalf of trainers and students. A main issue was that the overall time needed to complete a mission. The initially requested type of task had to cover actions on each step of the image exploitation process cycle, which potentially required several hours to complete. However, in the informal learning phase the game was one optional element of many. Thus, there was not enough time for students to play "just another mission" in addition to their regular exercises. Therefore, missions in the game need to evolve into smaller and more accessible tasks while keeping the major learning goals. In addition to this, the overall motivation to play the game and the retention rate should be improved further, as well as increasing the accessibility, meaning the game to evolve from a monolithic desktop application towards a web based and more mobile-like application. 
In conclusion, these new requirements and the results of the previous studies and evaluations implicated a need for adapting the initial game Lost Earth 2307 in terms of gameplay, motivation, usability and accessibility, which can be summarized as streamlining the game. Hence, this paper focuses on developing a concept for a streamlined version of the existing game, addressing the findings of the previous studies and the new requirements, while staying true to initial learning goals and to basic game elements. A preliminary analysis of the streamlined concept and a preliminary study on usability and immersion on a prototype are discussed and their implications on future developments are reviewed.

\section{METHOD \& IMPLEMENTATION}

\subsection{Method for Streamlining the Concept}

To address the new requirements and remaining a motivating, immersive game experience, while achieving the learning outcomes, the initial concept of Lost Earth 2307 was modified according to the new streamlined concept. (Yusoff et al. 2009) provide a framework for ensuring effective learning outcomes when creating serious games. The framework is derived from the input-process-outcome game model discussed by (Garris et al 2002) and presents several major components a serious game should consider (see Figure 1).

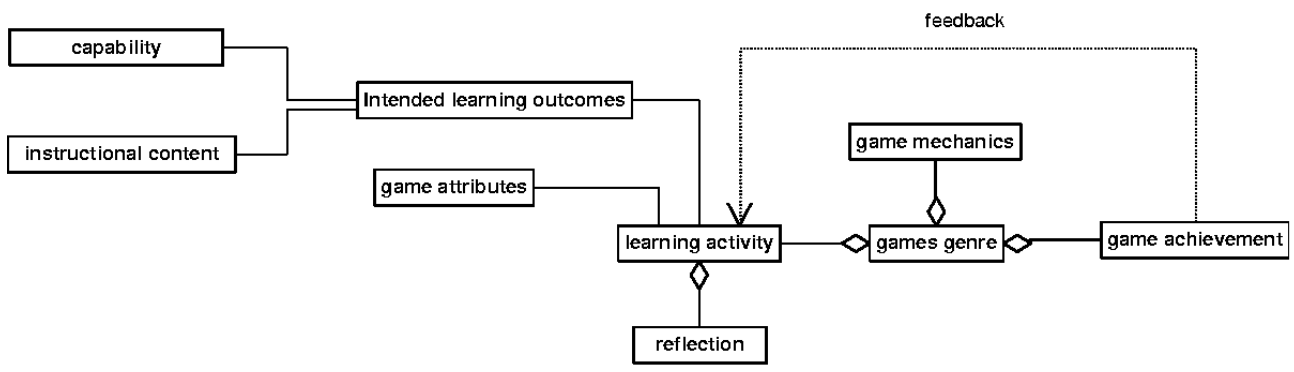

Figure 1. Yusoff et al. framework for serious games shown as a structural class diagram

An effective serious game should consider all components of the framework. A key component is the intended learning outcome. This is a combination of the intended capability and the instructional content (subject matter). Learning activity is key as well, because the activities within the game are what keeps players engaged and immersed. Along with the other components: reflection, game mechanics, game genre, game achievement, game attributes and a feedback loop an effective serious game is formed.

However, this method only shows whether all components are included in the serious game and gives an orientation, which attributes to develop. To analyze, whether the resulting serious game is motivating or not, the Octalysis Gamification Framework (Chou 2015) was used. It contains nine core drives to describe the source of engagement within the components and attributes of a gamification environment (see Figure 5, including results of the analysis).

\subsection{Implementation}

Streamlining the concept of Lost Earth 2307 followed Yusoff's serious game framework and Chou's Octalysis framework (Yusoff et al. 2009, Chou 2015). This chapter describes the main, streamlined elements and shows their contribution with regard to Yusoff.

A major streamlined element was replacing the initial task by smaller and quicker task types, thus by different types of missions. As stated before, a mission in the initial game always contained a complete image exploitation task, which generally took several hours to complete. Subsets of this process can now be asked using smaller tasks separately. For example, tasking of a sensor is now a stand-alone mission (was a mandatory part of the whole mission before) and labeling objects in imagery is now broken down into more suitable mission types such as multiple choice (see Figure 2 left), multiple response assessments and hot spot exercises (see Figure 2, right). All these new kinds of tasks allow players to perform a mission in a much shorter time than in the original game while still supporting the major learning objectives. 

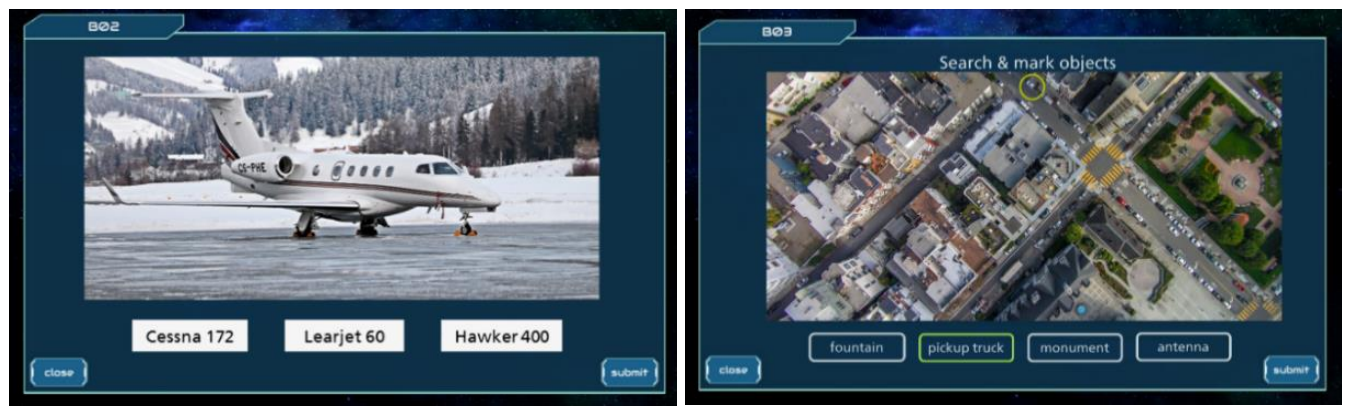

Figure 2. Examples for small tasks (left: multiple choice; right: hot spot)

The feedback loop after completing a mission was adapted respectively to the new task types. The new task types also lead to altering the mission sequence logic. This contributes to the game mechanics and feedback component of the serious game framework, whereas the new tasks itself contribute to the learning activity and to the need for quicker gameplay.
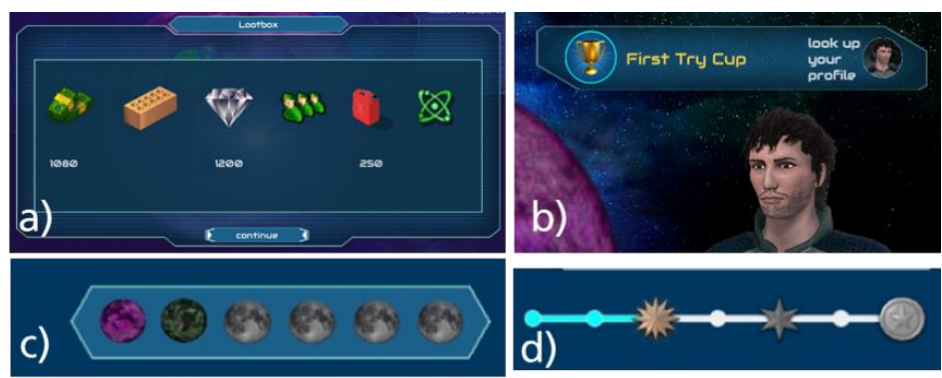

Figure. 3. Examples for achievements (a: loot box; b: trophy for completing first mission; $c$ : learning progress visualized by liberated colonies; $\mathrm{d}$ : career progress)

To increase motivation additionally, the game achievements were improved, as previous evaluations showed that additional rewarding elements would be beneficial for keeping players immersed. Hence, another streamlined element was introducing further achievement elements following ideas of (Zichermann and Cunningham 2011): Trophies as recognition for achievement, loot boxes for surprise gifting, visualization of the learning progress and enhanced scoring of a mission in form of an accumulated progress bar visualizing a career with milestones were implemented in the prototype (examples see Figure. 3). Associated to the achievements a new game mechanic was introdued, which is feeding Zichermanns ideas of collecting and customization: A player is now able to spend resources in order to enhance his personal room (see Figure 4).
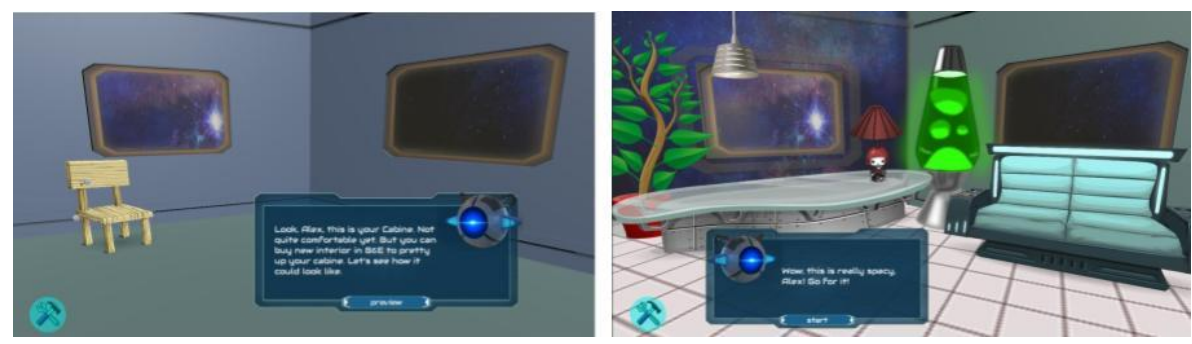

Figure. 4. Enhancing the personal room (left: initial state; right: fully enhanced)

These enhancements on the achievements contribute to Yusoff's game achievement and game mechanics components and specifically add to a more motivating gameplay.

To further address shorter gameplay and accessibility, some game mechanics were simplified. Especially the initially quite complex resource cycle was reduced to purchasing objects, which are required to start important missions. These simplifications correlate with adjustments to the general user interface. The user interaction is now designed to support gameplay via web and on mobile devices. 
From a technology perspective, the game engine was switched from Havok Vision to Unity. This allowed easy web based deployment as well as mobile deployment while allowing to port core code from the original game. This web based approach allows playing the game in a web browser instead of installing a desktop application which increases the requirement for improved accessibility.

\section{ANALYSIS AND PRELIMINARY STUDY}

\subsection{Analyzing the Streamlined Concept with Octalysis}

With the streamlined concept of Lost Earth 2307 considering all components of Yusoff's serious game framework and implementing presumably motivating elements, the next step was to analyze the prototype implementation in terms of fun and motivation using Chou's Octalysis framework (Chou 2015). Each of the eight core drives were reviewed and assigned relevant game elements, resulting in a score for each core drive (see Figure 5).

The general evaluation outcome of the Octalysis tool was "Proud of You / Good Motivation. [...]". Hence, the prototype implementation of the streamlined concept seems to be fun and motivating according to the Octalysis framework.

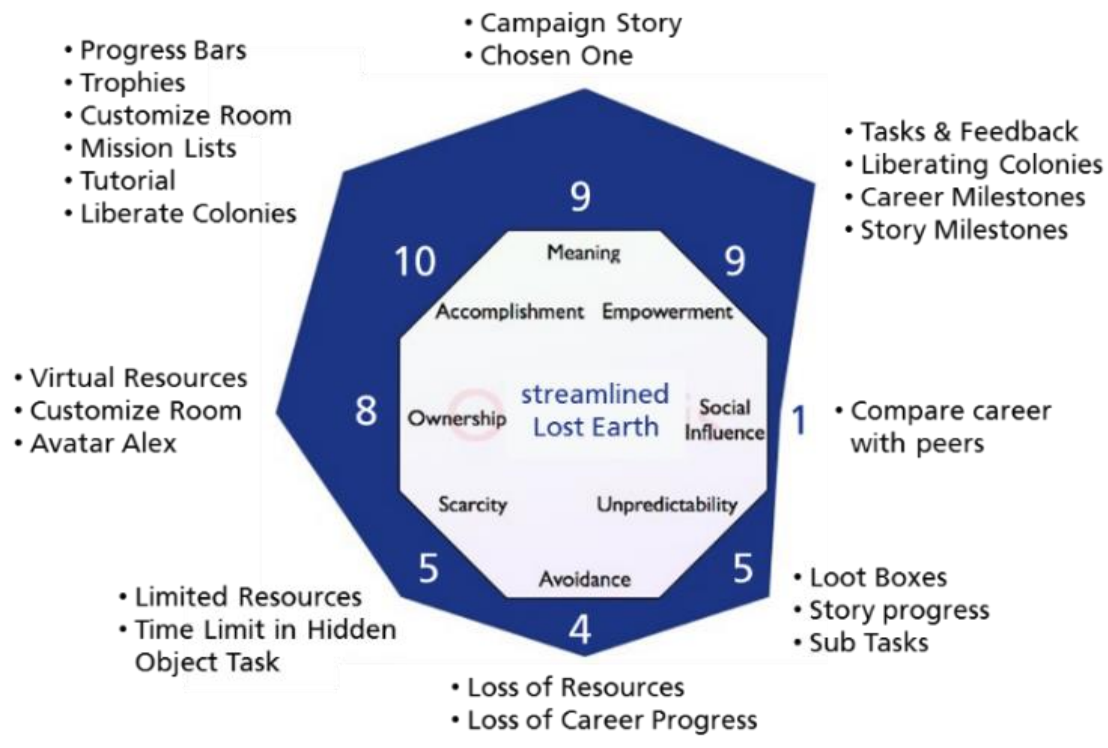

Figure 5. Result of Octalysis analyzation of the protoype implementation

\subsection{Setup of Preliminary Study}

The results of the Octalysis framework were positive on the one hand, but quite subjective on the other. In order to get a more objective opinion whether the prototype implementation of the concept is heading into the right direction in terms of immersion and usability, a preliminary study was conducted.

(Kannegieser et al. 2019) propose that experiencing flow state compares to experiencing the Total Immersion state of (Cheng et al. 2015). Thus, for measuring the state of immersion the Flow Short Scale questionnaire (FSS) by (Rheinberg et al. 2003) was used. Usability was evaluated using the System Usability Scale (SUS) questionnaire by (Brooke 1996).

The setup of the study followed five steps: First, introducing the participant to the procedure. Second, elicit general data (gender, gaming experience, etc.). Third, playing the prototype of the streamlined concept on a provided laptop, monitored as a 'Think Aloud Session', where participants were asked to verbalize what 
happens inside their head (Albert and Tullis 2013). Fourth, presenting the FSS questionnaire directly after playing the prototype to capture the first impressions of immersion and last but not least answering the SUS questionnaire.

Nine subjects participated in the study, three of whom were female and five of whom were male. The subjects were not members of the target group. However, as stated in a previous study, improving general usability and immersion is mostly independent of the initial target group (Atorf et al. 2019b). All subjects had gaming experience and mainly played games on desktop computers. The tasks were customized for the study so that non image exploitation experts were able to complete the missions.

\subsection{Results of the Preliminary Study}

Regarding the evaluation of the Flow Short Scale questionnaire, statements "3. I did not notice time passing" and "6. I am totally absorbed in what I am doing" showed interesting results (see Figure 6). Five subjects agreed that statement 3 does fully apply. For five subjects statement 6 was nearly true and for three subjects it did fully apply. For statement "5. My mind is completely clear" and for statement "7. The right thoughts/movements occur of their own accord." five subjects each affirmed level 5 on the Likert-scale.

All further statements of the Flow Short Scale varied widely in the results, so that no clear indications could be derived. However, the answers on statement 3 and 6 indicate, that some participants might have experienced a state of flow.

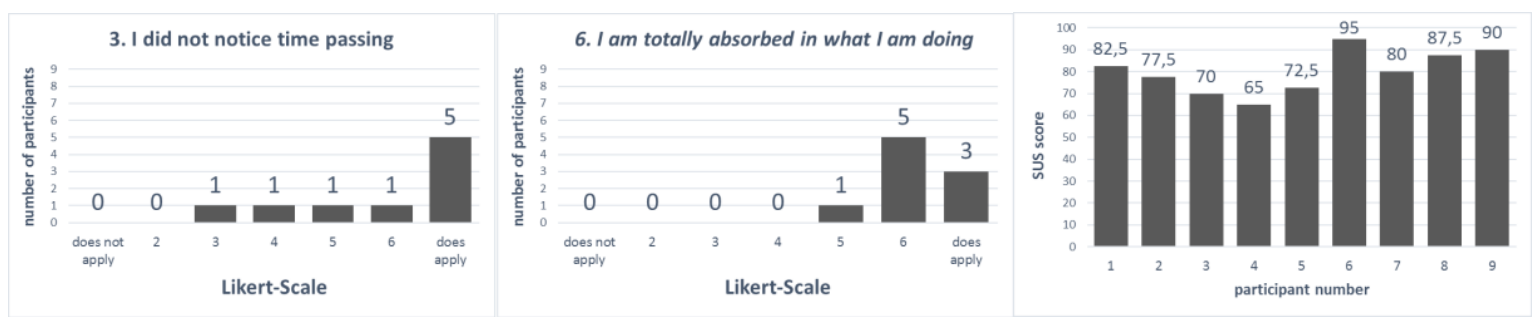

Figure 6. Evaluation of FSS questionnaire, statement three and six, as well as SUS questionnaire

Regarding usability, the highest SUS score was 95 and the lowest was 65 . A score of 68 is commonly accepted as at least "good" usability (Bangor et al. 2009). Thus, eight of the nine participants acknowledged good usability for the prototype (see Figure 6). Monitoring the 'Think Aloud Session' revealed specific hurdles while using the prototype. As an example, for some participants it was not clear enough how to exchange resources. However, unlike in the original game, no show-stopping usability issues were monitored. In addition, each SUS score in this preliminary study is better than the average SUS score of the original game, which was 50 (usability issues of original game and SUS scores see Atorf et al. 2019b).

Item 1 of the SUS questionnaire is "I think that I would like to use this game frequently.". This item is of interest for determining a player's motivation to return to the game. Six out of the nine participants agreed or strongly agreed to the statement of item 1 , although all participants were not members of the target group. This indicates, that some of these non-experts had fun playing the game and are willing to play again.

\subsection{Discussion}

The results of the Flow Short Scale questionnaire indicate that some participants might have experienced a state of flow. However, the number of subjects is far too low to make general assumptions. Furthermore, the gaming phase being limited to 20 minutes is creating a difficult environment for reaching a flow state at all. Hence, measuring flow as the optimum state of immersion seems not ideal within this setup and further evaluations should consider using the dedicated immersion questionnaires by (Cheng et al. 2015) to capture the level of immersion in more detail.

Regarding usability, the findings point towards an acceptable implementation for most of the participants. Nevertheless, the low number of subjects interdicts general conclusions. Nonetheless, some participants would like to play the game again. 


\section{CONCLUSION}

The paper presented a process on how to streamline the game design of an existing serious game and how the prototype implementation was evaluated by a preliminary study. At first, the initial game design was modified by addressing the findings of previous evaluations and following the serious game framework by Yusoff. The resulting streamlined concept was analyzed in terms of motivation and fun, using the Octalysis framework. A preliminary study evaluated usability and immersion of the prototype implementation. The findings indicated that the streamlined concept seems to be on the right track regarding motivation and usability. However, the study was preliminary and lacked a suitable number of participants.

In general, the presented method for streamlining an existing game design seems to work. Gameplay is shorter and more accessible in comparison to the original game implementation, because of the improved SUS score and because of using a web based deployment. Future work will focus on improvements to usability and motivational elements, based on the findings of the preliminary study. These further improvements will be evaluated subsequently in qualified studies.

\section{REFERENCES}

Albert, W., Tullis, T., 2013. Measuring the user experience: collecting, analyzing, and presenting usability metrics. Morgan Kaufmann, Waltham.

Atorf D. et al, 2019a. Balancing Realism and Engagement for a Serious Game in the Domain of Remote Sensing. In Games and Learning Alliance, Proceedings of the 7th International Conference, GALA 2018, pp.146-156.

Atorf D. et al, 2019b. Study on Enhancing Learnability of a Serious Game by Implementing a Pedagogical Agent. In Games and Learning Alliance, Proceedings of the 8th International Conference, GALA 2019, pp.158-168. Springer Nature $\mathrm{CH}$.

Bangor, A. et al. 2009. Determining What Individual SUS Scores Mean: Adding an Adjective Rating Scale. In Journal of Usability Studies, Volume 4, Issue 3, pp. 114-123.

Bopp, M. 2006. Immersive Didaktik und Framingprozesse in Computerspielen. In Neitzel, B. and Nohr, R.F. Das Spiel mit dem Medium. Partizipation-Immersion-Interaktion. Zur Teilhabe an den Medien von Kunst bis Computerspiel, pp. 170-186, Schüren, Marburg.

Brooke, J. 1996. SUS - A quick and dirty usability scale. In Usability Evaluation in Industry. Routledge.

Cheng, M.-T. et al. 2015. Game Immersion Experience: Its Hierarchical Structure and Impact on Game-based Science Learning. In Journal of Computer Assisted Learning, Vol 31, Issue 3, pp. 232-253.

Chou, Y. 2015. Actionable Gamification: Beyond Points, Badges, and Leaderboards. California, Octalysis Media.

Garris, R. et al. 2002. Games, Motivation, and Learning: A Research and Practice Model, In Simulation \& Gaming, vol. 33, pp. 441-467.

Kannegieser, E. et al. 2019. Conducting an Experiment for Validating the Combined Model of Immersion and Flow. In Proceedings of the 11th International Conference on Computer Supported Education, Vol 2, pp. 252-259.

Prensky, M. 2007. Digital Game-Based Learning. Paragon House, St. Paul.

Rheinberg, F. et al. 2003. Die Erfassung des Flow-Erlebens. In Diagnostik von Motivation und Selbstkonzept. Göttingen: Hogrefe, pp. 261-279.

Yusoff, A. et al. 2009. A Conceptual Framework for Serious Games. In 2009 Ninth IEEE International Conference on Advanced Learning Technologies, pp. 21-23.

Zichermann, G., Cunningham, C. 2011. Gamification by Design: Implementing Game Mechanics in Web and Mobile Apps (1st ed.). O'Reilly Media, Sebastopol, California. 